\title{
Multivariate matrix-exponential distributions
}

\author{
Mogens Bladt* and Bo Friis Nielsen ${ }^{\dagger}$
}

February 18, 2008

\section{Introduction}

In this extended abstract we define a class of distributions which we shall refer to as multivariate matrix-exponential distributions (MVME). They are defined in a natural way, inspired by the definition of univariate matrixexponential distributions, as the distributions on $\mathbb{R}_{+}^{n}$ having a rational (multidimensional) Laplace transform. A multidimensional rational function is the fraction between two multidimensional polynomials. The marginal distributions are hence univariate matrix-exponential distributions and in general dependent. In one dimension, matrix-exponential distributions are defined as distributions on $\mathbb{R}_{+}$with a rational Laplace transform, which in turn is equivalent to its density being a weighted sum of the elements of a matrixexponential. Thereby their name.

The main purpose of this work is to characterize the MVME distributions in terms of one-dimensional matrix-exponential distributions. In Section 2 we provide some background on univariate matrix-exponential distributions as well as a historical review of two subclasses of MVME which were defined previously by respectively Assaf et al. and Kulkarni. We also formulate Kulkarni's definition in terms of the structure of their projections which is more in line with our characterization theorem. The main result states that a multivariate distribution is a MVME if and only if any non-negative nonnull linear combination of the coordinates are again matrix-exponential. This

*Institute for Applied Mathematics and Systems, National University of Mexico, Mexico City, bladt@stats.iimas.unam.mx

$\dagger$ Informatics and Mathematical Modelling, Technical University of Denmark, Kgs. Lyngby, bfn@imm.dtu.dk

Dagstuhl Seminar Proceedings 07461

Numerical Methods for Structured Markov Chains http://drops.dagstuhl.de/opus/volltexte/2008/1397 
theorem is stated in section 3. We conclude the paper with a construction (in Section 4) and a number of examples (in Section 5), illustrating that the approach taken in this paper can be used to unify and understand a number of distributions discussed previously.

\section{Preliminaries}

In this section we first provide some necessary background from the theory of one-dimensional matrix-exponential distributions. In the second part of the section we review two classes of multivariate phase-type distributions, which are special cases of the MVME distributions. In particular, the class proposed by Kulkarni Kulkarni (1989) is of interest, and we provide a characterization of this subclass in terms of the structure of the intensity matrices of the linear combinations of the coordinates which is generalizable to MVME.

\subsection{Univariate matrix-exponential distributions}

Definition 2.1 A non-negative random variable $X$ is said to have a matrixexponential distribution if the Laplace transform $L(s)=\mathbb{E}[\exp (-s X)]$ is a rational function in $s$.

The following result is standard see e.g. Asmussen \& Bladt (1997) page 315 for a proof.

Lemma 1 A random variable is matrix-exponentially distributed if and only if there exists a triple $(\boldsymbol{\beta}, D, \mathbf{d})$ such that the density $f(x)$ of $X$ can be expressed as

$$
f(x)=\boldsymbol{\beta} e^{D x} \mathbf{d} .
$$

Here $\boldsymbol{\beta}$ and $\mathbf{d}$ is a row respectively column vector of dimension $m$ and $D$ is a $m \times m$ matrix, possibly with complex elements.

The triple $(\boldsymbol{\beta}, D, \mathbf{d})$ is called a representation of the matrix exponential distribution. The Laplace transform of $X$ can be determined from a representation $(\boldsymbol{\beta}, D, \mathbf{d})$ as

$$
L(s)=\boldsymbol{\beta}(s I-D)^{-1} \mathbf{d},
$$

where $I$ is the identity matrix of dimension $m$. Any matrix-exponential distribution has infinitely many representations. The dimension of $D$ is called 
the order of the representation. If the rational function $L(s)$ of Equation (1) cannot be reduced we say that $m$ is also the degree of the distribution (Asmussen \& O'Cinneide (2000)).

The non-centralized moments of a matrix-exponentially distributed random variable is easily derived by successive differentiation in the Laplace transform.

$$
M_{i}=\mathbb{E}\left(X^{i}\right)=i ! \boldsymbol{\beta}(-D)^{-(i+1)} \mathbf{d} \quad i=0,1, \ldots .
$$

We shall also need what has been termed reduced moments,

$$
\mu_{i}=\frac{M_{i}}{i !}, \quad i=0,1, \ldots
$$

\subsection{Two classes of multivariate phase-type distribu- tions}

Assaf et al. (1984) introduced the class of multivariate phase-type distributions MPH by considering a special type of reward structure on a onedimensional phase-type representation. Consider a generator $D$ of dimension $m$ and a $n$-dimensional vector $\mathbf{X}$ of random variables. With each random variable is associated a set $\Gamma_{i}$ of termination states. Whenever, $\Gamma_{i}$ is entered that set can not be left. The random variable $X_{i}$ is defined as the exit time of $\Gamma_{i}^{c}$. Although this definition seems restrictive surprisingly many of the distributions, that has been proposed as multivariate exponential and gamma distributions are actually of this type. The class is sufficiently restricted that it is possible to give closed form expressions for the joint density and Laplace transform of $\mathbf{X}$.

A rephrasing of the definition of MPH says that reward for $X_{k}$ is cumulated with rate 1 in states belonging to $\Gamma_{k}^{c}$, where $\Gamma_{k}^{c}$ is the complement of $\Gamma_{k}$. Based on this interpretation Kulkarni (1989) introduced the class MPH* as a generalization of the MPH class. In the class $\mathrm{MPH}^{*}$ reward for $X_{i}$ is accumulated in state $j$ with rate $K_{i j}$. There is no restriction on the phase-type generator $D$. If the total sojourn time in state $j$ of this Markov chain before absorption is denoted by $Y_{j}$ we have that the $n$ dimensional random vector $\mathbf{X}$ is defined element wise by $X_{i}=\sum_{j=1}^{m} K_{i j} Y_{j}$. The class proposed by Kulkarni and its natural generalization to the matrix-exponential case is surprisingly rich. The following theorem which gives an alternative characterization in terms of all non-negative projections was not given explicitly by Kulkarni. We state it without proof. 
Theorem 2.1 A distribution in $M P H^{*}$ is uniquely determined by the representation $(\boldsymbol{\alpha}, T(\mathbf{a}))$ of the Phase type distributed random variable $\langle\mathbf{a}, \mathbf{X}>$ with $T(\mathbf{a})=\Delta(K \mathbf{a})^{-1} T$.

\section{Multivariate matrix-exponential distribu- tions}

We define multivariate matrix-exponential distributions as a natural extension of the univariate case.

Definition 3.1 A non-negative random vector $\mathbf{X}=\left(X_{1}, \ldots, X_{n}\right)$ of dimension $n$ is said to have multivariate matrix-exponential distribution (MVME) if the joint Laplace transform $L(\mathbf{s})=\mathbb{E}[\exp (-<\mathbf{s}, \mathbf{X}>)]$ is a multi-dimensional rational function, that is, a fraction between two multi-dimensional polynomials. Here $<\cdot, \cdot>$ denotes the inner product in $\mathbb{R}^{n}$ with $\mathbf{s}=\left(s_{1}, \ldots, s_{n}\right)$.

Our main theorem characterizes the class of MVME.

Theorem 3.1 A vector $\mathbf{X}=\left(X_{1}, \ldots, X_{n}\right)$ follows a multivariate matrixexponential distribution if and only if $\langle\mathbf{a}, \mathbf{X}\rangle=\sum_{i=1}^{n} a_{i} X_{i}$ has a univariate matrix-exponential distribution for all non-negative vectors $\mathbf{a} \neq \mathbf{0}$.

Proof: In the full version of the paper we prove the main result by means of continued fractions. We note that there is an alternative approach to this using the Caley Hamilton theorem. However, we currently prefer the approach using continued fractions.

The following corollary is immediate.

Corollary 3.1 Let $\mathbf{X}=\left(X_{1}, \ldots, X_{n}\right)$ have a MVME distribution and let $\mathbf{A}$ be a non-negative $m \times n$ matrix. Then $\mathbf{Y}=\mathbf{A X}$ has a MVME distribution. In particular, all marginal distributions are again matrix-exponentially distributed.

The next theorem gives some further structure to the MVME class. 
Theorem 3.2 Let $\mathbf{X}=\left(X_{1}, \ldots, X_{n}\right)$ follow a multivariate matrix-exponential distribution Then we may write its moment generating function for $\langle\mathbf{a}, \mathbf{X}>$ as

$$
\frac{f_{1}^{*}(\mathbf{a}) s^{m-1}+f_{2}^{*}(\mathbf{a}) s^{m-2}+\ldots+f_{m-1}^{*}(\mathbf{a}) s+1}{g_{0}^{*}(\mathbf{a}) s^{m}+g_{1}^{*}(\mathbf{a}) s^{m-1}+\ldots+g_{m-1}^{*}(\mathbf{a}) s+1}
$$

where the terms $f_{i}^{*}(\mathbf{a})$ and $g_{i}^{*}(\mathbf{a})$ are sums of monomials in $\mathbf{a}$ of order $m-i$.

Inspired by Theorem 3.1 we propose the following definition of a multivariate phase-type distribution.

Definition $3.2 A$ vector $\mathbf{X}=\left(X_{1}, \ldots, X_{n}\right)$ has a multivariate phase-type distribution (MVPH) if $<\mathbf{a}, \mathbf{X}>$ has a (univariate) phase-type distribution for all non-negative $\mathbf{a} \neq \mathbf{0}$.

The following definition is now natural.

Definition 3.3 Let $M M E^{*}$ be the subclass of $M V M E$, where $<\mathbf{a}, \mathbf{X}>$ can be expressed by a representation with constant initial vector and generator matrix of the form $(\Delta(K \mathbf{a}))^{-1} T$ by $M M E^{*}$

The class $\mathrm{MME}^{*}$ contains most but probably not all multivariate distributions with rational Laplace transforms discussed to date.

Theorem 3.3 An MVME with distribution with $m=2$ will always have at least one representation in $M M E^{*}$.

However, cases exist where it is not possible to find a MME* representation corresponding to the the degree of $\langle\mathbf{a}, \mathbf{X}\rangle$.

Theorem 3.4 There exists MVME distributions where the MVME order is strictly less than the $M M E^{*}$ order.

Proof: The proof is based on the non-existence of a three dimensional MME* representation of Krishnamoorthy and Parthasarathy's Multivariate Exponential when $n=3$. For a discussion of this distribution see section 48.3.3 in Kotz, Balakrishnan \& Johnson (2000). 


\subsection{Joint distribution of age and residual life in a re- newal process}

Theorem 3.5 Let $A_{t}$ and $R_{t}$ be the age and residual life time in a stationary renewal process respectively with interarrival time distribution $f(x)$. The joint distribution of $A_{t}$ and $R_{t}$ is given by $\frac{f(x+y)}{\int_{0}^{\infty} x f(x) d x}$.

Proof: The joint distribution of $\left(A_{t}, R_{t}\right)$ in a renewal process is given by

$$
P\left(R_{t} \geq x, A_{t}<y\right)=\int_{t-y}^{t}(1-F(t+x-u)) \mathrm{d} U(u)
$$

see e.g. Yanushkevichius (1995). Inserting $1 / \mathbb{E} X$ for the stationary renewal density and differentating we obtain the result.

Theorem 3.6 In a stationary renewal process with matrix-exponentially distributed inter-arrival times with representation $(\boldsymbol{\alpha}, C)$ the joint distribution of age and residual life is a $M M E^{*}$ with representation

$$
\left(\left(\frac{\boldsymbol{\alpha}(-C)^{-1}}{\mu}, \mathbf{0}\right),\left[\begin{array}{cc}
C & -C \\
0 & C
\end{array}\right],\left[\begin{array}{cc}
\mathbf{e}_{m} & \mathbf{0} \\
\mathbf{0} & \mathbf{e}_{m}
\end{array}\right]\right)
$$

where $m$ is the dimension of $C$.

Proof: The proof is purely analytical.

\section{Bivariate Morgenstern type distributions}

In this section we discuss the Farlie-Gumbel-Morgenstern construction, which will indeed lead to a rational Laplace transform and hence a MVME distribution whenever the marginal distributions are ME. Let $F_{i}(x), i=1,2$ 
be univariate distributions and define the joint distribution in terms of the Farlie-Gumbel-Morgenstern copula to be

$$
F\left(x_{1}, x_{2}\right)=F_{1}\left(x_{1}\right) F_{2}\left(x_{2}\right)\left(1+\rho\left(1-F_{1}\left(x_{1}\right)\right)\left(1-F_{2}\left(x_{2}\right)\right)\right) \quad .
$$

These distributions are in $\mathrm{MME}^{*}$ if $F_{1}(x), F_{2}(x)$ have rational Laplace transforms. To see this we start with the following general result.

Lemma 2 Let $F_{i}^{\min }(x)=1-\left(1-F_{i}(x)\right)^{2}$ and $F_{i}^{\max }(x)=F_{i}^{2}(x)$ such that $F_{i}^{\min }(x)$ and $F_{i}^{\max }(x)$ are cumulative distribution functions of minimum respectively maximum of two independent random variables distributed according to $F_{i}(x)$. Then the bivariate Morgenstern distribution $F\left(x_{1}, x_{2}\right)$ from $F_{1}\left(x_{1}\right) F_{2}\left(x_{2}\right)$ is

$$
\begin{aligned}
F\left(x_{1}, x_{2}\right)= & \frac{1+\rho}{4} F_{1}^{\max }\left(x_{1}\right) F_{2}^{\max }\left(x_{2}\right)+\frac{1-\rho}{4} F_{1}^{\max }\left(x_{1}\right) F_{2}^{\min }\left(x_{2}\right)+ \\
& \frac{1-\rho}{4} F_{1}^{\min }\left(x_{1}\right) F_{2}^{\max }\left(x_{2}\right)+\frac{1+\rho}{4} F_{1}^{\min }\left(x_{1}\right) F_{2}^{\min }\left(x_{2}\right)
\end{aligned}
$$

Proof: The result is proved by eliminating $F_{i}(x)$ using $F_{i}(x)=\frac{F_{i}^{\min }(x)+F_{i}^{\max }(x)}{2}$ in $(2)$.

The lemma is useful as we can express the Morgenstern type distributions as a non-negative mixture of four bivariate distributions. Each of these four terms are distributions of independent variables formed from the two basic distributions $F_{1}(x)$ and $F_{2}(x)$. The following lemma is well-known for phase-type distributions.

Lemma 3 Let $X_{1}$ and $X_{2}$ be two independent matrix-exponentially distributed random variables with representation $(\boldsymbol{\alpha}, T)$. Then $\max \left(X_{1}, X_{2}\right)$ is matrix-exponentially distributed with representation

$$
\left((\boldsymbol{\alpha} \otimes \boldsymbol{\alpha}, \mathbf{0}),\left[\begin{array}{cc}
T \oplus T & (\mathbf{t} \oplus \mathbf{t}) \mathbf{e} \\
0 & T
\end{array}\right]\right)
$$

and $\min \left(X_{1}, X_{2}\right)$ is matrix-exponentially distributed with representation

$$
(\boldsymbol{\alpha} \otimes \boldsymbol{\alpha}, T \oplus T)
$$


The next lemma is also a generalization of the phase-type case.

Lemma 4 A matrix-exponential distribution with representation $(\boldsymbol{\alpha}, T)$ can alternatively be expressed by the representation $(\tilde{\boldsymbol{\alpha}}, \tilde{T})$ where

$$
\tilde{T}=\Delta(\boldsymbol{\pi})^{-1} T^{\prime} \Delta(\boldsymbol{\pi}), \tilde{\boldsymbol{\alpha}}=\frac{\boldsymbol{\pi} \bullet \mathbf{t}}{\boldsymbol{\pi t}}
$$

Here $\mathbf{t}=-T \mathbf{t}$ and $\boldsymbol{\pi}$ is a positive solution to $\boldsymbol{\pi}(T+\mathbf{t} \boldsymbol{\alpha})=\mathbf{0}$.

Proof: The result is proved by direct verification.

Theorem 4.1 Consider the bivariate Farlie-Gumbel-Morgenstern distribution formed from two matrix-exponential distribution is in $M M E^{*}$ with marginal representation of $F_{i}$ given by $\left(\boldsymbol{\alpha}_{i}, S_{i}\right), i=1,2$. A $M M E^{*}$ representation $(\boldsymbol{\alpha}, S, K)$ is

$$
\begin{gathered}
\boldsymbol{\alpha}=\left(\boldsymbol{\alpha}_{1} \otimes \boldsymbol{\alpha}_{1}, \mathbf{0}, \mathbf{0}, \mathbf{0}\right) \\
S=\left[\begin{array}{cccc}
S_{1} \oplus S_{1} & \frac{1}{2}\left(\mathbf{s}_{1} \oplus \mathbf{s}_{1}\right) & \frac{1-\rho}{4}\left(\mathbf{s}_{1} \oplus \mathbf{s}_{1}\right) \mathbf{e} \tilde{\boldsymbol{\alpha}}_{2}^{(M, m)} & \frac{1+\rho}{4}\left(\mathbf{s}_{1} \oplus \mathbf{s}_{1}\right) \mathbf{e} \tilde{\boldsymbol{\alpha}}_{2}^{(m)} \\
0 & S_{1} & \frac{1+\rho}{2} \mathbf{s}_{1} \tilde{\boldsymbol{\alpha}}_{2}^{(M, m)} & \frac{1-\rho}{2} \mathbf{s}_{1} \tilde{\boldsymbol{\alpha}}_{2}^{(m)} \\
0 & 0 & \Delta_{1}^{-1} S_{2}^{T} \Delta_{1} & \Delta_{1}^{-1}\left(\mathbf{s}_{2} \oplus \mathbf{s}_{2}\right)^{T} \Delta_{2} \\
0 & 0 & 0 & \tilde{S}_{2}^{(m)}
\end{array}\right], \\
K=\left[\begin{array}{cc}
\mathbf{e}_{m_{1}} \otimes \mathbf{e}_{m_{1}} & 0 \\
\mathbf{e}_{m_{1}} & 0 \\
0 & \mathbf{e}_{m_{2}} \otimes \mathbf{e}_{m_{2}} \\
0 & \mathbf{e}_{m_{2}}
\end{array}\right]
\end{gathered}
$$

with

$$
\begin{gathered}
\Delta_{1}=\Delta\left(\boldsymbol{\pi}_{2}^{(M, m)}\right), \quad \Delta_{2}=\Delta\left(\boldsymbol{\pi}_{2}^{(m)}\right) \\
\boldsymbol{\pi}_{2}=\mu_{2}^{-1} \boldsymbol{\alpha}_{2}\left(-S_{2}\right)^{-1}, \quad \tilde{\boldsymbol{\alpha}}_{2}=\mu_{2}^{-1} \boldsymbol{\pi}_{2} \bullet \mathbf{s}_{2}, \\
\boldsymbol{\pi}_{2}^{(m)}=\left(\mu_{2}^{(m)}\right)^{-1}\left(\boldsymbol{\alpha}_{2} \otimes \boldsymbol{\alpha}_{2}\right)\left(-S_{2} \oplus S_{2}\right)^{-1}, \quad \tilde{\boldsymbol{\alpha}}_{2}^{(m)}=\left(\mu_{2}^{(m)}\right)^{-1} \boldsymbol{\pi}_{2}^{(m)} \bullet\left(\mathbf{s}_{2} \oplus \mathbf{s}_{2}\right)
\end{gathered}
$$




$$
\begin{aligned}
& \boldsymbol{\pi}_{2}^{(M)}=\left(\frac{\mu_{2}^{(m)}}{\mu_{2}^{(M)}} \boldsymbol{\pi}_{2}^{(m)}, \boldsymbol{\pi}_{2}^{(M, m)}\right) \quad, \quad \tilde{\boldsymbol{\alpha}}_{2}^{(M)}=\left(\mu_{2}^{(M)}\right)^{-1}\left(\mathbf{0}, \boldsymbol{\pi}_{2}^{(M, m)} \bullet \mathbf{s}_{2}\right) \\
& \tilde{S}_{2}^{(m)}=\Delta\left(\boldsymbol{\pi}_{2}^{(m)}\right)^{-1}\left(S_{2} \oplus S_{2}\right)^{T} \Delta\left(\boldsymbol{\pi}_{2}^{(m)}\right) \\
& \tilde{S}_{2}^{(M)}=\Delta\left(\boldsymbol{\pi}_{2}^{(M)}\right)^{-1}\left[\begin{array}{cc}
\left(S_{2} \oplus S_{2}\right)^{T} & 0 \\
\left(\mathbf{s}_{2} \oplus \mathbf{s}_{2}\right)^{T} & S_{2}^{T}
\end{array}\right] \Delta\left(\boldsymbol{\pi}_{2}^{(M)}\right) \\
& =\left[\begin{array}{cc}
\Delta\left(\boldsymbol{\pi}_{2}^{(M, m)}\right)^{-1} \stackrel{\tilde{S}_{2}^{(m)}}{\left(\mathbf{s}_{2} \oplus \mathbf{s}_{2}\right)^{T} \Delta\left(\boldsymbol{\pi}_{2}^{(m)}\right)} & \Delta\left(\boldsymbol{\pi}_{2}^{(M, m)}\right)^{-1} S_{2}^{T} \Delta\left(\boldsymbol{\pi}_{2}^{(M, m)}\right)
\end{array}\right]
\end{aligned}
$$

Proof: The result can be proved by calculation of the Laplace transforms.

The representation of Theorem 4.1 can be motivated by a probabilistic argument in the phase-type case.

\section{$5 \mathrm{MPH}^{*}$ representations for multivariate ex- ponential and gamma distributions}

This section discusses a number of bivariate and multivariate exponential and gamma distributions that have been discussed in the literature. Many of these have rational Laplace transform and most of those with rational Laplace transform are in the $\mathrm{MPH}^{*}$ or even in the MPH class. In general the multivariate gamma distributions belong to MVME whenever the shape parameters are integer. The main reference is Kotz, Balakrishnan \& Johnson (2000). In this reference the distributions are classified as bivariate and multivariate exponential respectively gamma distributions. Here we take the approach of classifying according to the $(\boldsymbol{\alpha}, T, K)$ representations. The treatment here is non-exhaustive. However, we believe that it should be sufficiently complete to illustrate how these various results can be unified and how the underlying generating probabilistic mechanism can be understood by using the terminology of MVME and MME*. 


\subsection{The case where states can be revisited}

The form that should contain all distributions of this section is

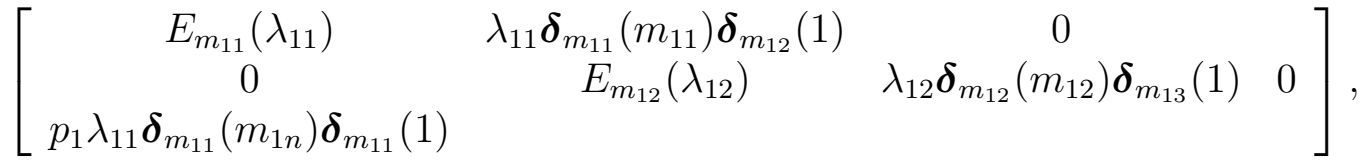

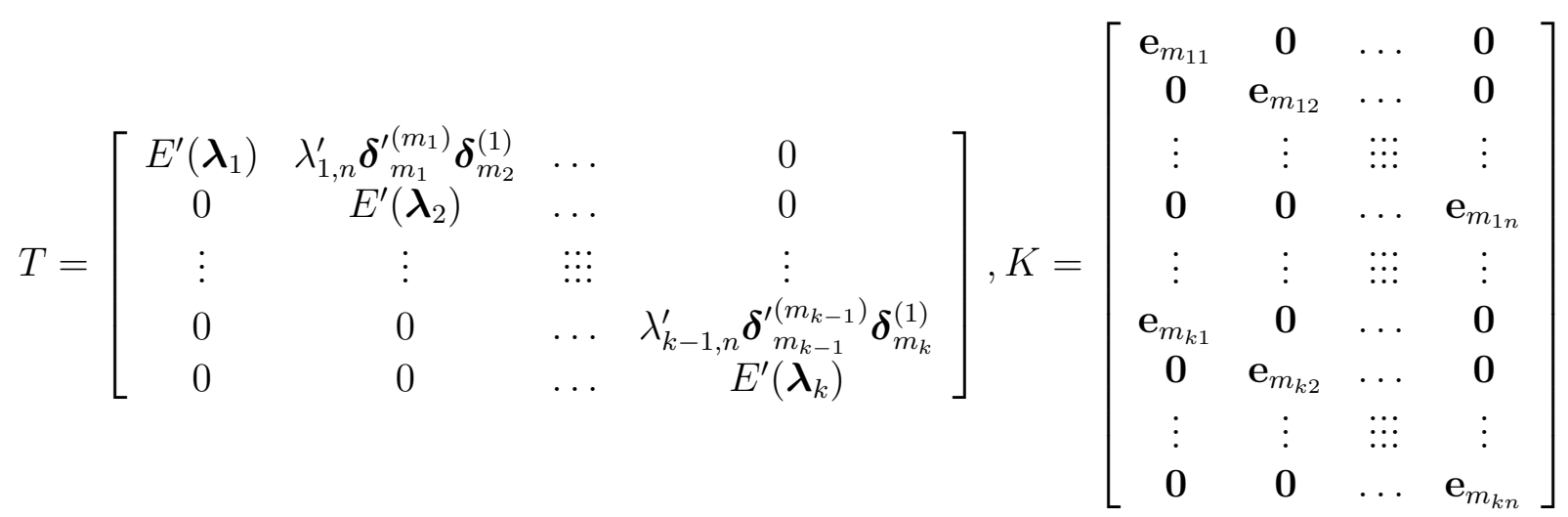

where $E_{n}(\lambda)$ denotes a sub-generator of Erlang form of dimension $n, \boldsymbol{\delta}_{i}$ is a vector with one in the $i$ th position and zeros elsewhere, $m_{i}=\sum m_{i j}, m=$ $\sum m_{i}, \boldsymbol{\lambda}_{i}=\left(\lambda_{i 1} \mathbf{e}_{m_{i, 1}}^{\prime}, \ldots, \lambda_{i, n} \mathbf{e}_{m i, n}^{\prime}\right), \lambda_{j k}^{\prime}=\lambda_{j k} p_{j}$, and $E^{\prime}\left(\boldsymbol{\lambda}_{j}\right)=E\left(\boldsymbol{\lambda}_{j}\right)+$ $p_{j} \lambda_{j, n} \boldsymbol{\delta}_{m_{j}}^{\left(m_{j}\right)} \boldsymbol{\delta}_{m_{j}}^{(1)}$.

For Kibble and Moran's Bivariate Gamma 48.2.3 we have $n=2, k=$ $1, p=, \lambda_{1,1}=\lambda_{1,2}$. The distribution is described in Kotz, Balakrishnan \& Johnson (2000) Section 2.3 pp.436-437. Gaver's Multivariate Gamma 48.3.2 is a variant of the KMBG, For Moran and Downton's Multivariate Exponential 47.3.6 we have $k=1$, while for Moran and Downton's Bivariate Exponential 47.2.7 we further have $n=2$

\subsection{The case where states cannot be revisited}

\section{Cheriyan and Ramabhadran's multivariate Gamma 48.3.1}

For integer valued shape parameters the distributions are in MME*.

$$
f\left(x_{1}, \ldots, x_{n}\right)=\frac{e^{-\sum_{i=1}^{n} x_{i}}}{\prod_{i=0}^{n}\left(m_{i}-1\right) !} \int_{0}^{\min \left(x_{i}\right)} y^{m_{0}-1}\left(\prod_{i=1}^{n}\left(x_{i}-y\right)^{m_{i}-1}\right) e^{(n-1) y} \mathrm{~d} y
$$


$\gamma=\delta_{1}$

$$
T=E_{\left(\sum_{i=0}^{n} m_{i}\right)} \quad K=\left[\begin{array}{cccc}
\mathbf{e}_{m_{0}} & \mathbf{e}_{m_{0}} & \ldots & \mathbf{e}_{m_{0}} \\
\mathbf{e}_{m_{1}} & \mathbf{0} & \ldots & \mathbf{0} \\
\mathbf{0} & \mathbf{e}_{m_{2}} & \ldots & \mathbf{0} \\
\vdots & \vdots & \ldots: \vdots & \ldots \\
\mathbf{0} & \mathbf{0} & \ldots & \mathbf{e}_{m_{n}}
\end{array}\right]
$$

Cheriyan and Ramabhadran's bivariate Gamma distribution is described in Section 2.2 of Kotz, Balakrishnan \& Johnson (2000) 48.2.2. The distribution is obtained from the multivariate case by closing $k=2$. McKay's Bivariate Gamma 48.2.1 distribution in described in Section 2.1 of Kotz, Balakrishnan \& Johnson (2000). The distribution is the special case of Cheriyan and Ramabhadran's bivariate Gamma with $m_{2}=0$.

\section{The Marshall-Olkin family}

A number of distribution classes are either subsets of the Multivariate Marshall and Olkin's Exponential 47.3.2 or closely related. We state the joint survival function

$$
\begin{aligned}
\bar{F}_{X_{1}, \ldots, X_{n}}\left(x_{1}, \ldots, x_{n}\right)= & \exp \left(-\sum_{i=1}^{n} \lambda_{i} x_{i}-\sum_{i_{1}<i_{2}} \lambda_{i_{1} i_{2}} \max \left(x_{i_{1}}, x_{i_{2}}\right)\right. \\
& \left.-\sum_{i_{1}<i_{2}<i_{3}} \lambda_{i_{1} i_{2} i_{3}} \max \left(x_{i_{1}}, x_{i_{2}}, x_{i_{3}}\right) \cdots-\lambda_{12 \ldots n} \max \left(x_{1}, \ldots, x_{n}\right)\right) .
\end{aligned}
$$

These distributions are in $\mathrm{MPH}^{*}$. A representation for $n=3$ is $\gamma=$ $(1,0,0,0,0,0,0)$

$$
T=\left[\begin{array}{ccccccc}
-\tau_{1} & \lambda_{3} & \lambda_{2} & \lambda_{1} & \lambda_{23} & \lambda_{13} & \lambda_{12} \\
0 & -\tau_{2} & 0 & 0 & \lambda_{2} & \lambda_{1} & 0 \\
0 & 0 & -\tau_{3} & 0 & \lambda_{3} & 0 & \lambda_{1} \\
0 & 0 & 0 & -\tau_{4} & 0 & \lambda_{3} & \lambda_{2} \\
0 & 0 & 0 & 0 & -\lambda_{1} & 0 & 0 \\
0 & 0 & 0 & 0 & 0 & -\lambda_{2} & 0 \\
0 & 0 & 0 & 0 & 0 & 0 & -\lambda_{3}
\end{array}\right] \quad K=\left[\begin{array}{ccc}
1 & 1 & 1 \\
1 & 1 & 0 \\
1 & 0 & 1 \\
0 & 1 & 1 \\
1 & 0 & 0 \\
0 & 1 & 0 \\
0 & 0 & 1
\end{array}\right]
$$

Here we have taken $\tau_{1}=\lambda_{1}+\lambda_{2}+\lambda_{3}+\lambda_{12}+\lambda_{13}+\lambda_{23}+\lambda_{123}, \tau 2=\lambda_{1}+\lambda_{2}+$ $\lambda_{12}, \tau_{3}=\lambda_{1}+\lambda_{3}+\lambda_{13}$, and $\tau_{4}=\lambda_{2}+\lambda_{3}+\lambda_{23}$. 
Among related distributions are Freund's Multivariate Exponential 47.3.1, Freund's Bivariate Exponential 47.2.3, Block and Basu's Multivariate Exponential 47.3.3, Olkin and Tong's Multivariate Exponential 47.3.4, Raftery's Multivariate Exponential 47.3.7, Raftery's Bivariate Exponential 47.2.9, Friday and Patil's Bivariate Exponential 47.2.5, Prèkopa and Szàntai's Multivariate Gamma 48.3.4, and Prèkopa and Szàntai's Bivariate Gamma 48.2.8.

\subsection{Joint distribution of MAP/RAP inter arrival times}

The joint distribution of a finite number of sojourn times in a Markovian Arrival Process (MAP, see e.g. Lucantoni, Meier-Hellstern \& Neuts (1990)) is contained in $\mathrm{MPH}^{*}$. If a MAP parameterized by the matrices $\left(D_{0}, D_{1}\right)$ and with initial phase or state distribution $\boldsymbol{\theta}$, then the first two sojourn times $X_{1}$ and $X_{2}$ have joint density

$$
f\left(x_{1}, x_{2}\right)=\boldsymbol{\theta} e^{D_{0} x_{1}} D_{1} e^{D_{0} x_{2}} D_{1} \mathbf{e} .
$$

The $\mathrm{MME}^{*}$ representation is $\boldsymbol{\gamma}=(\boldsymbol{\theta}, \mathbf{0})$,

$$
T=\left[\begin{array}{cc}
D_{0} & D_{1} \\
0 & D_{0}
\end{array}\right], \quad K=\left[\begin{array}{cc}
\mathbf{e}_{m} & \mathbf{0} \\
\mathbf{0} & \mathbf{e}_{m}
\end{array}\right] .
$$

Indeed the same expressions are valid for the more general class of Rational Arrival Processes (RAP, see Asmussen \& Bladt (1999)).

\subsection{Restricted support}

For most if not all distributions discussed in the literature the support of at least one variable will be all positive reals regardless of the value of the other variable. However, it is easy to construct distributions without this restriction. We can write

$$
\left[\begin{array}{l}
X_{1} \\
X_{2}
\end{array}\right]=\left[\begin{array}{cc}
\frac{2}{3} & \frac{1}{3} \\
\frac{1}{3} & \frac{2}{3}
\end{array}\right]\left[\begin{array}{l}
Z_{1} \\
Z_{2}
\end{array}\right] \Leftrightarrow\left[\begin{array}{l}
Z_{1} \\
Z_{2}
\end{array}\right]=\left[\begin{array}{cc}
2 & -1 \\
-1 & 2
\end{array}\right]\left[\begin{array}{l}
X_{1} \\
X_{2}
\end{array}\right],
$$

where $\left(Z_{1}, Z_{2}\right)$ are independent $\exp (1)$ distributed random variables. By a standard formula for transformation of random variables we find the joint density of $\left(X_{1}, X_{2}\right)$

$$
f\left(x_{1}, x_{2}\right)=f_{Z_{1}}\left(2 x_{1}-x_{2}\right) f_{Z_{2}}\left(-x_{1}+2 x_{2}\right)=e^{-\left(x_{1}+x_{2}\right)}, \quad \frac{1}{2} x_{1} \leq x_{2} \leq 2 x_{1} .
$$




\section{References}

Asmussen, S. \& Bladt, M. (1997). "Renewal Theory and Queueing Algorithms for Matrix-Exponential Distributions". In Alfa, A. \& Chakravarthy, S. R., editors, Matrix-analytic methods in stochastic models, pages 313-341.

Asmussen, S. \& Bladt, M. (1999). "Point processes with finite-dimensional probabilities". Stochastic Processes and their Applications, 82(1):127142.

Asmussen, S. \& O'Cinneide, C. A. (2000). Matrix-exponential distributions, pages 435-440. Encyclopedia of Statistical Sciences, Supplementary Volume. John Wiley \& Sons, Inc.

Assaf, D.; Langberg, N. A.; Savits, T. H. \& Shaked, M. (1984). "Multivariate Phase-Type Distributions". Operations Research, 32(3):688-702.

Kotz, S.; Balakrishnan, N. \& Johnson, N. L. (2000). Continuous Multivariate Distributions. John Wiley and Sons.

Kulkarni, V. G. (1989). "A new class of multivariate phase type distributions". Operations Research, 37(1):151-158.

Lucantoni, D. M.; Meier-Hellstern, K. S. \& Neuts, M. F. (1990). "A SingleServer Queue with Server Vacations and a Class of Non-Renewal Arrival Processes". Adv.Apl.Prob., 22:676-705.

Yanushkevichius, R. (1995). "Stability of one characterization by the properties of the renewal process". Lithuanian Mathematical Journal, 35(2):158-162. 